\title{
BDA leads call for plasma donations to fight COVID-19
}

The British Dental Association (BDA) is urging dentists and dental team members in England who have tested positive for COVID-19 to put themselves forward as potential donors for the NHS convalescent plasma programme, currently being used in clinical trials to improve the recovery of people with the virus.

Leading the call is Birmingham dentist and newly elected BDA Chair, Eddie Crouch, who developed COVID-19 during lockdown, has fully recovered and has volunteered for the programme.

Baseline figures from a large-scale study at Birmingham University - covering over 1,500 dental team members across the Midlands - showed $16 \%$ of dentists, hygienists, therapists and nurses had COVID-19 antibodies prior to the resumption of face-to-face care on 8 June using enhanced PPE and new safety protocols. This compared to between 6-10\% for the general population in the region.

Donated plasma is being transfused into people who are still unwell, and the antibodies could save lives, with the number of cases receiving donations quadrupling in the last month.

To sign up just visit the NHSBT's website (https://bit.ly/3e5ol3e). Donors need to:

- Have had coronavirus (either a positive test or symptoms)

- Be over 17

- Be able to travel to one of the donation centres (https://bit.ly/34tJNM0).

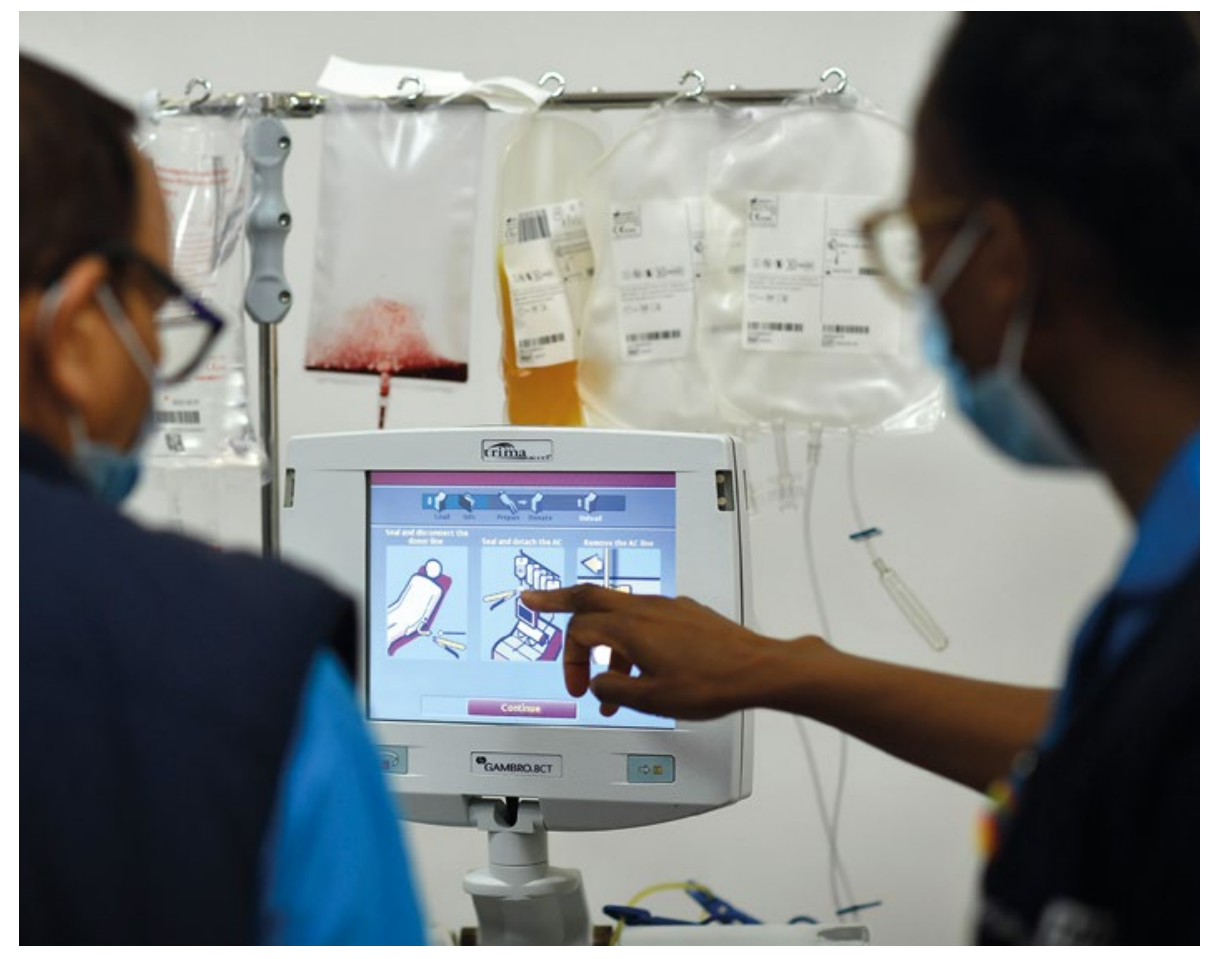

The BDA has written to all its members in England encouraging those who meet the criteria to volunteer. The programme is particularly interested in hearing from men, people aged over 35 , people from Asian communities, and people who were treated in hospital. These people are more likely to have higher levels of antibodies in their plasma which can help fight infection.

BDA Chair Eddie Crouch said: 'COVID-

19 is with us for the foreseeable future. As a healthcare professional I hope my own misfortune can now help patients in desperate need.

'Dentists and their teams have gone over and above during the pandemic, manning urgent dental care centres, redeployed to the front line, and working all out to restore services. For those who have tested COVIDpositive these plasma donations can now save lives.

'I hope that any colleagues ready and willing to go that extra mile will consider putting themselves forward.'

\section{New salivary gland organs discovered}

By Manas Dave, Manchester, UK

The salivary gland system has three paired major glands with numerous minor glands spread through the aerodigestive tract. A team of researchers, led by Dr Valstar in the Netherlands, have reported that they have discovered a new pair of salivary glands in the posterior nasopharynx. Their research has been published in the journal Radiotherapy \& Oncology. ${ }^{1}$

Visualisation of salivary glands can be achieved with positive emission tomography/computed tomography (PET/CT) with radio-labelled ligands for the prostate-specific membrane antigen
(PSMA). Surprisingly, the researchers observed structures in the nasopharynx demonstrating ligand uptake similar to salivary glands during patient PSMA PET/ CT scans. These structures did not fit any known previous anatomical descriptions. The researchers investigated this by evaluating 100 consecutively scanned prostate/paraurethral cancer patients. All patients had the same PSMA-positive area bilaterally in their posterior nasopharynx. Moreover, two human cadaver dissections were performed and the area of interest analysed through histology. Microscopic examination confirmed mucous gland tissue and draining ducts. As these glands are situated over the torus tubarius, they were named 'tubarial glands'.

It remains to be seen if the glands are classified as minor or major salivary glands but the discovery of a new body structure in 2020 certainly sparks scientific interest.

\section{Reference}

1. Valstar M H, de Bakker B S, Steenbakkers R J H M et al. The tubarial salivary glands: a potential new organ at risk for radiotherapy. Radiother Oncol 2020; doi: 10.1016/j.radonc.2020.09.034. Online ahead of print. 